\title{
Atenção em saúde mental em municípios de pequeno porte
}

\author{
Rafaela Silva Amorim Suzarte Ferreira* \\ Helena Moraes Cortes** \\ Paula Hayasi Pinho***
}

\begin{abstract}
Resumo
Este trabalho teve como objetivo verificar os principais desafios e dificuldades da Atenção à Saúde Mental em municípios de pequeno porte I. Trata-se de uma revisão narrativa da literatura, realizada na Biblioteca Virtual em Saúde (BVS-BIREME) em abril de 2018, com os descritores: saúde mental e municípios de pequeno porte considerando os últimos 10 anos (2007-2017). Além dos artigos foi utilizado um livro de relevância no tema abordado. Os resultados evidenciaram desconhecimento dos profissionais da rede sobre a Reforma Psiquiátrica e a falta de trabalhadores qualificados, desde a atenção básica à gestão municipal. Este artigo destaca a necessidade de discussões relevantes na área. campo da Saúde Mental em pequenos municípios, a fim de facilitar o debate sobre a Reforma Psiquiátrica, seus dispositivos e a reorganização da RAPS.

Palavras-chave: Saúde Mental e Municípios de pequeno porte I
\end{abstract}

\section{Mental health care in small municipalities}

\begin{abstract}
This work aimed to verify the main challenges and difficulties of Mental Health Care in small municipalities I. This is a narrative review of the literature, carried out in the Virtual Health Library (VHL-BIREME) in April 2018, with the descriptors: mental health and small municipalities considering the last 10 years (2007-2017). In addition to the articles, a book of relevance in the topic was used. The results evidenced a lack of knowledge of the network professionals about the Psychiatric Reform and the lack of skilled workers, from basic care to municipal management. This article highlights the need for relevant discussions in the field of Mental Health in small municipalities I, in order to facilitate the debate about the Psychiatric Reform, its devices and the reorganization of RAPS.

Key words: Mental Health; Mental Health Services; Health Unic System.
\end{abstract}

* Psicóloga. Especialista em Gestão em Saúde. Centro de Ciências da Saúde. Universidade Federal do Recôncavo da Bahia.

** Doutora em Ciências pela EEUSP. Professora Assistente I do curso de Enfermagem - Centro de Ciências da Saúde. Universidade Federal do Recôncavo da Bahia

*** Doutora em Ciências pela EEUSP. Professora Assistente I do curso de Medicina - Centro de Ciências da Saúde. Universidade Federal do Recôncavo da Bahia 


\section{Introdução}

O tratamento da loucura, até pouco tempo, era marcado por um modelo hospitalocêntrico, excludente, estigmatizador, reforçando o lema "aos loucos... o hospício!”. A forma de vivenciar a condição humana estabeleceu que historicamente, pessoas com transtornos mentais devessem ser presas em cadeias públicas e, excluídas do convívio social, o que isentaria de ameaças a sociedade (Foucault, 2005).

De acordo com Foucault (2005), com o passar do tempo e buscando tornar mínimo o sofrimento da pessoa acometida pelo transtorno mental, emergiram mudanças evidenciadas no modelo de cuidado, baseado na cidadania, na liberdade e na reinserção social destes portadores de sofrimento psíquico. Foi nesta perspectiva que, Phillippe Pinel, considerado o pai da psiquiatria, na França, teve a oportunidade de libertar os acorrentados - assim eram conhecidos os indivíduos doentes psiquicamente. Desta forma, os asilos então foram substituídos pelos manicômios. Portanto, "uma espécie de instância perpétua de julgamento: o louco tinha que ser vigiado nos seus gestos, rebaixado nas suas pretensões, contradito no seu delírio, ridicularizado nos seus erros [...]" (Foucault, 2000, p.82).

No Brasil, somente no século XX, a partir da década de 70 , houve a grande demarcação para impulsionar o redirecionamento no que diz respeito aos cuidados com a loucura (Amarante, 2007). Essa movimentação aconteceu atrelada à Reforma Sanitária, sendo o marco primordial à garantia da proteção e dos direitos das pessoas com transtornos mentais instituída na Lei 10.216/01, que redireciona a Política de Assistência à Saúde Mental no país (Brasil, 2002).

A Reforma Psiquiátrica trouxe mudanças no paradigma da atenção e gestão nas práticas e, em defesa dos direitos da pessoa com transtorno mental. A reforma psiquiátrica possibilitou aos estados e municípios implementar uma política de saúde mental pautada no princípio da equidade e da reinserção social (Paiano, Maftum, Haddad \& Marcon (2016). Apesar das dificuldades enfrentadas pelo sistema de saúde pública no Brasil, é notório que, a Reforma Psiquiátrica brasileira trouxe um olhar diferente no que tange ao cuidado de pessoas com transtornos mentais.

Nada obstante, a partir da implementação da nova proposta, pouco se conhece sobre o funcionamento da Política de Assistência à Saúde Mental na maioria dos municípios, em especial os de pequeno porte I. Nestes, considerando o cenário nacional, os gestores atuam de forma descomprometida com a melhoria das condições de vida da população (Luzio \& L'Abbate, 2009).

Conforme o Instituto Brasileiro de Geografia e Estatística - IBGE (2010), o Brasil tem 5.570 municípios e um Distrito Federal. Desses, 3.914 são municípios de pequeno porte I (são considerados municípios de porte I aqueles com dados populacionais até 20.000 mil habitantes). Contudo, 17,1\% vivem em municípios com população abaixo de 20 mil habitantes, ou seja, aproximadamente com 32 milhões de pessoas (IBGE,2010).

É importante complementar que os municípios de pequeno porte I, geralmente são localizados em áreas geográficas mais distantes e, com situações de maior vulnerabilidade e risco social, gerenciando uma escassez de recursos financeiros para ser aplicado na atenção básica, tornando ainda mais delicado o financiamento do tratamento em saúde mental (Mendonça, Mattos, Oliveira, Domingos, Okamura, Carvalho \& Nunes, 2016).

A implantação da RP brasileira direcionou a substituição do modelo hospitalocêntrico para um modelo comunitário de saúde mental, a ser implementado nos municípios, como os Centros de Atenção Psicossocial CAPS (Silva \& Campos, 2015). O trabalho desenvolvido no CAPS tem como objetivo promover o resgate da cidadania e a reintegração social dos usuários de saúde mental na comunidade, além de trabalhar na perspectiva da reabilitação psicossocial.

Os municípios de pequeno porte, com população acima de 15.000 mil habitantes, podem solicitar ao Ministério da Saúde a implantação do CAPS I, sendo caso aceito, custeado com subsídio federal e contrapartida do município (Brasil, 2011).

No que concerne ao financiamento destes municípios que tem o CAPS I, recebem uma quantia mensal de $\mathrm{R} \$ 28.305,00$ (vinte e oito mil e trezentos e cinco reais) para custeio das ações de atenção psicossocial. Nas situações em que há repasse mensal maior do que o valor mencionado, conforme a portaria, deverá haver avaliação in loco das condições de estrutura, equipe e produção e repactuação para adequação dos valores repassados (Brasil, 2011).

A partir da pesquisa na literatura nacional foram encontrados poucos estudos que tratam da atenção em saúde mental nos municípios de pequeno porte I. Neste sentido, este estudo teve o objetivo de compreender quais os principais desafios/dificuldades da assistência em saúde mental nos municípios de pequeno porte I.

A Política Nacional de Saúde Mental prevê que os serviços de saúde de municípios de pequeno 
porte I, devem estar inseridos na Rede de Atenção Psicossocial(RAPS), o que parece ser um desafio significativo a ser superado no campo das políticas públicas brasileiras.

Assim teve-se como questão de pesquisa: quais os principais desafios/dificuldades da assistência em saúde mental nos municípios de pequeno porte I?

\section{Método}

Trata-se de um estudo de revisão narrativa. Vosgerau e Romanowsk (2014) definem que este tipo de revisão não estabelece uma metodologia rigorosa e replicável em termos de reprodução de dados e, permite uma análise ampla da literatura.

A busca foi realizada na Biblioteca Virtual de Saúde (BVS-BIREME) e ocorreu em abril de 2018, com as seguintes palavras chaves: "saúde mental" e municípios de pequeno porte I" tendo como período de referência os últimos 10 anos (2007 a 2017). Foram considerados livros e capítulos de relevância para o tema.

Os critérios de inclusão foram: artigos científicos disponíveis na íntegra em meio eletrônico, de livre acesso e redigidos na língua portuguesa. Os critérios de exclusão foram: teses e anais de congressos ou conferências e publicações duplicadas.

Durante a análise dos artigos iniciou-se a leitura dos títulos, seguida da leitura dos resumos e, posteriormente, dos textos completos e posteriormente confrontou-se com a literatura.

\section{Resultados e discussão}

A atenção psicossocial discutida nos estudos de Boarini (2009), que buscou conhecer a atenção em saúde mental na rede pública de saúde em um município de pequeno porte; de Silva, Martins, Maia, Oliveira e Souza (2014), que conheceu a experiência de familiares de pessoas com transtorno mental em municípios de pequeno porte e, de Pessoa Junior, Miranda, Melo e Silva (2013) que descreveram a experiência de gestão em saúde mental na atenção primária em um município de pequeno porte; destacaram-se enquanto estudos que desvelaram modelos de cuidado de caráter comunitário que consideraram a pessoa, o contexto, a família, a rede e o próprio profissional, e que visaram desta forma, devolver a cidadania às pessoas em sofrimento psíquico, em municípios de pequeno porte.

Assim, a rede de cuidado torna-se primordial e, deve estar atrelada à ideia de modificação do paradigma da atenção em Saúde Mental, cujas ações direcionam para o campo teórico, ético, social, político e técnico (Luzio \& L'Abbate, 2009).

Nesta perspectiva, é importante pensar na atenção psicossocial não como um modelo ou sistema, mas como um processo em construção, disposto a romper barreiras e, aprender a partir das mudanças que surgirão por tentativa e erro (Amarante, 2007).

Observou-se nos artigos analisados, que os atores envolvidos no campo da Saúde Mental em municípios pequenos, englobou uma gama de sujeitos como os profissionais que atuam na Atenção Primária à Saúde (APS), nos ambulatórios e nos CAPS; além dos gestores de saúde municipais, os usuários e familiares destes serviços.

Segundo Paiano, Maftum, Haddad e Marcon (2016), geralmente, a pessoa em sofrimento psíquico busca a atenção primária à saúde (APS) como porta de entrada para seu tratamento na Unidade Básica de Saúde (UBS). Todavia, muitas vezes os profissionais a encaminham para o atendimento no CAPS e nos ambulatórios, como se os casos de transtornos mentais leves não fossem responsabilidade da APS, desconsiderando o sujeito e suas necessidades globais, o estigmatizando como doente mental (Paiano, Maftum, Haddad \& Marcon, 2016). Isso pode demonstrar a falta de entendimento da Política Nacional de Saúde Mental por parte de profissionais e até da gestão, além de trazer indícios do desconhecimento da RP brasileira que deve acontecer também em municípios pequenos (Brasil, 1992).

O desenvolvimento das ações voltadas à saúde mental, precisa do diálogo entre os profissionais dos diferentes níveis de atenção. Boarini (2009) aponta que os profissionais da UBS utilizam a medicalização como solução encontrada para auxiliar as pessoas com transtornos mentais, devido à falta de uma equipe especializada e a ascensão deste tipo de demanda, ocorre a repetição das receitas médicas a partir da consulta com o psiquiatra.

Autores vêm discutindo a atuação centrada na perspectiva médica e, restrita à prescrição de medicamentos, visto que isto, por si só, não contribui para potencializar a autonomia no cotidiano do usuário (Rosa \& Zambenedetti, 2014).

Ressalta-se que os profissionais dos CAPS's têm a missão de fazer o matriciamento de casos de saúde mental das UBS's, realizando o diálogo entre os níveis de complexidade da rede de saúde e, evitando os encaminhamentos para a rede especializada (Quinderé, Jorge \& Franco, 2014).

Desse modo, os serviços de atenção psicossocial precisam desenvolver no território suas peculiaridades e, 
possibilitar o resgate de habilidades sociais e, construção de vínculos nos serviços da rede (Amarante, 2007). Um estudo num serviço residencial terapêutico, no município de São Paulo, mostrou a forte relação deste serviço com os demais equipamentos da rede, configurando-se como uma excelente estratégia de reabilitação psicossocial no campo da Reforma Psiquiátrica Brasileira (Cortes \& Barros, 2017).

Os profissionais dos CAPS's precisam criar vínculos também com os familiares dos usuários e, considerar que o conviver com um familiar com transtorno mental também pode gerar sobrecarga e adoecimento para o cuidador, pois há comportamentos e sintomas que se arrastam, desequilibram, e dificultam a relação familiar (Silva, Martins, Maia, Oliveira \& Souza, 2014). Oferecer atendimento aos familiares, ainda é um dos grandes desafios em municípios pequenos, o que acaba por potencializar o sentimento de abandono destes familiares, visto a ausência de uma rede de serviços estruturada que, possa auxiliar no cuidado e que dê suporte a essa tarefa de cuidar (Duarte \& Neto, 2016).

A inexistência de uma rede de atenção à saúde mental traz como consequência o aumento de surtos psicóticos, suicídios, depressão - além de outros transtornos - evidenciando a falta de cuidados em saúde mental que resultam em uma comunidade adoecida. Uma rede funciona com o trabalho das equipes de saúde, articulando entre si as distintas instâncias dentro do sistema, tendo uma significativa expressão no meio social (Quinderé, Jorge \& Franco, 2014).

Nesta linha de pensamento, a RAPS que é responsável por articular a "teia da saúde mental" nos municípios, aposta na intersetorialidade para a continuidade da construção da atenção em saúde mental. A atenção em saúde mental se organiza a partir de uma rede que, geralmente nos municípios, é proveniente da articulação do gestor de saúde (Silva \& Campos, 2015).

A gestão inadequada se reflete na ausência da Atenção Psicossocial que implica, diretamente, na repetição do modelo psiquiátrico pautado na centralidade, sendo o médico o profissional mais procurado. Diante disso, a atenção é voltada para o cuidado biológico enfraquecendo as outras possibilidades terapêuticas e a própria RAPS, conforme discute Silva, Martins, Maia, Oliveira \& Souza (2014).

Em municípios pequenos autores observaram que ao invés de uma rede, há alguns pontos de atenção, os quais nem sempre se apresentam de modo articulado (Rosa \& Zambenedetti, 2014).
Embora a política nacional de saúde mental venha desconstruindo paradigmas e, formando um novo redirecionamento na prática com o sujeito em sofrimento psíquico, ainda se percebe que em municípios pequenos, a práxis executada está atrelada aos resquícios da história da loucura. "Os novos serviços necessitam de profissionais capacitados para operar dentro de novas estratégias" (Hirdes, 2009).

Entretanto, há falta de conhecimento sobre os dispositivos em saúde mental dos municípios pequenos, referidos na pesquisa de Silva, Martins, Maia, Oliveira e Souza (2014), persiste e implica diretamente na atuação dos profissionais de saúde que dificultam o acesso da população ao serviço. Corroborando com o estudo de Alvarenga e Garcia (2009) que, evidenciam que técnicos com diferentes formações, capacitação e infraestrutura física nos serviços comunitários de saúde mental é um dos desafios para a implementação da Política de Saúde Mental em municípios pequenos.

Entende-se que, a educação permanente é de extrema importância para os profissionais desenvolverem boas práticas em Saúde Mental, além de possibilitar a produção de conhecimento e reflexões acerca de possíveis mudanças para a atuação do trabalhador e seu processo de trabalho. Desse modo, oportuniza a construção de ações inovadoras para os serviços em saúde (Marcolino, Fantinatti \& Gozzi, 2018).

Levando em consideração que, a saúde mental em municípios pequenos faz parte de um bloco da saúde municipal e, recebe seus recursos em um conjunto destinado à saúde, há a possibilidade da não utilização destes recursos na saúde mental, assim é fundamental que o gestor tenha conhecimento da Reforma Psiquiátrica, para que possibilite o avanço na implementação da política de saúde mental no município (Silva \& Campos, 2015). Muitas vezes, a falta de um gestor comprometido impossibilita a manutenção dos serviços em saúde mental, exceto o CAPS que tem financiamento assegurado (Brasil, 2011).

Conforme postula Alvarenga e Garcia (2009) é fundamental que o município não busque apenas a ampliação de serviços extra-hospitalares, mas também incentive dentro da comunidade, o acontecimento de campanhas com o objetivo de desconstruir o status de periculosidade associado à loucura, e sobretudo modifique a ideia de exclusão como única alternativa de tratar os sujeitos com algum transtorno mental.

Muitas vezes, uma alternativa possível para conseguir operar mudanças na realidade dos municípios é a implementação da ferramenta do Planejamento Estraté- 
gico Situacional (PES), construído a partir da matriz de programação de ações que possibilita ao município traçar os problemas e propor estratégias de enfrentamento da problemática a fim de melhorias no âmbito da saúde mental (Kleba, Krauser \& Vendruscolo, 2011).

Para refletir: "a revisão de toda legislação é um primeiro aspecto, pois tanto o código penal quanto o civil ou ainda outras leis e normas sociais estão repletos de referências nocivas aos sujeitos em sofrimento psíquico e representam obstáculos significativos ao exercício da cidadania" (Amarante, 2007, p. 69).

Partindo deste pressuposto, as dificuldades e limitações devem ultrapassar as barreiras e possibilitar novos saberes e redirecionamento da saúde mental em municípios de pequeno porte.

\section{Conclusões}

Este estudo fomenta a necessidade de discussões pertinentes em torno da Política Nacional de Saúde Mental em municípios de pequeno porte I.

Pode-se afirmar que, uma das maiores dificuldades refere-se ao fato de a gestão de saúde municipal ser descomprometida com a saúde mental. Tal descomprometimento reflete-se diretamente na atenção à saúde mental nos diferentes dispositivos da rede, como o CAPS's, os ambulatórios e as Unidades Básicas de Saúde.

Vale salientar que, a condução da assistência em saúde mental, em municípios pequeno porte I, geralmente sofre modificações quando existe a troca de gestão, pois esta interfere no financiamento direcionado para a área da saúde mental e pode impedir a reformulação dos serviços.

Outros dois desafios/ dificuldades encontradas nos municípios de pequeno porte sugerem a ausência de atendimento aos familiares dos usuários de saúde mental, e a ausência da implementação da própria Rede de Atenção Psicossocial.

A fim de superar os desafios/dificuldades da gestão em saúde mental, em municípios de pequeno porte, pode-se pensar nas seguintes estratégias: gestão municipal comprometida com a especificidade da saúde mental; promoção do diálogo entre os profissionais nos diferentes níveis de atenção; fortalecimento das redes de cuidado; reorganização da RAPS de forma articulada e compartilhada com outros municípios e na lógica da regionalização da saúde; educação permanente para os trabalhadores com foco na reabilitação psicossocial; (re) financiamento das ações e serviços de forma planejada, sobretudo, para os CAPS que precisam ter protagonismo na atenção psicossocial, construindo vínculos na rede e, evitando as internações psiquiátricas, muito comum em municípios de pequeno porte.

Em síntese, faz necessário discussões em relação à assistência em saúde mental por gestores, profissionais, comunidades e pessoas acometidas por transtornos mentais, a fim de possibilitar mudanças no paradigma de exclusão social que envolve a pessoa com transtorno mental ainda vigente em nosso meio social. As pessoas com transtornos mentais devem ser assistidas em seus municípios de referência, nos seus territórios geográficos e existenciais, com ações compartilhadas tanto na APS, quanto na atenção especializada.

\section{Referências}

ALVARENGA, M. S. \& GARCIA, M. L. T.(2009). A implementação da política de saúde mental em município de pequeno porte - o caso de São José do Calçado/ ES - Brasil. Emancipação, Ponta Grossa, 9(2), 181-195.

Amarante, P. (2007). Saúd Mental e Atenção Psicossocial. Rio de Janeiro: Editora Fiocruz.

Brasil (1992). Portaria SAS/MS n 224, de 29 de janeiro de 1992 - Estabelece diretrizes e normas para o atendimento em saúde mental. Brasília: Ministério da Saúde.

(2002). Portaria n 336, de 19 de fevereiro de 2002 -Dispõe sobre a proteção e os direitos das pessoas portadoras de transtornos mentais e redireciona o modelo assistencial em saúde mental. Brasília: Ministério da Saúde.

(2011). Portaria/GM n. ${ }^{\circ}$ 3.088, de 23 de dezembro de 2011 - Institui a Rede de Atenção Psicossocial para pessoas com sofrimento ou transtorno mental e com necessidades decorrentes do uso de crack, álcool e outras drogas, no âmbito do SUS. Brasília: Ministério da Saúde.

(2011). Portaria no 3.089, de 23 de dezembro de 2011- Dispõe, no âmbito da Rede de Atenção Psicossocial, sobre o financiamento dos Centros de Atenção Psicossocial (CAPS). Brasília: Ministério da Saúde.

BOARINI, M. L.(2009). A (desconhecida) reforma psiquiátrica em municípios aquém de pequeno porte. Psicologia em Revista, 15(1), 28-50. Belo Horizonte.

CORTES, H. M., \& BARROS, S. (2017). Reabilitação psicossocial de moradores de um serviço residencial terapêutico. Journal of Nursing and Health, 7(2), 148-63.

DUARTE, L. G. M. F., NETO, F., \& LEITE, J. (2016). Familiares responsáveis pelo cuidado de pessoa com transtorno mental em um município de pequeno porte. Pesquisas e Práticas Psicossociais, 11(2), 473-488.

FOUCAULT, M. (2005). História da Loucura: na Idade Clássica/ (J. T. Coelho Neto, trad.). São Paulo: Perspectiva.

(2000)Doença Mental e Psicologia. Rio de Janeiro: Tempo Brasileiro.

HIRDES, A.(2009). A reforma psiquiátrica no Brasil: uma (re) visão. Ciência ¿ Saúde Coletiva, 14(1), 297-305.

IBGE - Instituto Brasileiro de Geografia e Estatísticas.(2010. Recuperado a partir de: http://cidades.ibge.gov.br/xtras/perfil.php?codmun=292850.

KLEBA, M. E., KRAUSER, I.M. \& VENDRUSCOLO, C. (2011). O Planejamento Estratégico Situacional no ensino da gestão em Saúde da Família. Texto Contexto Enferm, 20(1), 184-93. Florianópolis.

LUZIO, C.A. \& L'ABBATE, S. A.(2009) atenção em saúde mental em municípios de pequeno e médio portes: ressonâncias da reforma psiquiátrica. Ciênc Saúde Coletiva, 14(1),105-16.

MARCOLINO, T. Q., FANTINATTI, E. N. \& GOZZI, A. P. N. F. (2018), Comunidade de prática e cuidado em saúde mental: uma revisão sistemática. Trab. educ. saúde, Rio de Janeiro, Recuperado a partir de:http://www. scielo.br/scielo.php?script $=$ sci_arttext\&pid $=$ S1981-77462018005001104\&ln $\mathrm{g}=$ pt\&nrm=isso.

MENDONÇA, F.F., MATTOS, L. F. A., OLIVEIRA, E. B. D., DOMINGOS, C. M., OKAMURA, C. T., CARVALHO, B. G. \& NUNES, E. F. P. A.(2016). Participação dos municípios de pequeno porte no Projeto Mais Médicos 
para o Brasil na macrorregião norte do Paraná. Ciência \& Saúde Coletiva, 21(9),2907-2915.

PAIANO, M., MAFTUM, M. A., HADDAD, M. C. L. \& MARCON, S. S. (2016). Ambulatório de saúde mental: fragilidades apontadas por profissionais. Texto Contexto Enferm, 25(3),e0040014.

PESSOA JUNIOR, J. M., MIRANDA, F. A. N., MELO, R. M. \& SILVA, M. B. (2013). Gestão e Saúde Mental em um Município de Pequeno Porte: uma experiência em um município de pequeno porte. Rev. APS, 16(3), 328-332.

QUiNDERÉ, P. H. D., JORGE, M. S. B. \& FRANCO, T. B. (2014). Rede de Atenção Psicossocial: qual o lugar da saúde mental? Physis Revista de Saúde Coletiva, 24 (1), 253-271. Rio de Janeiro.

ROSA, M. P. \& ZAMBENEDETTI, G. (2014) Descompassos da Reforma Psiquiátrica: A Saúde Mental em um município do Interior do Paraná. Desafios: Revista Interdisciplinar da Universidade Federal do Tocantins, 1(1), 317-335.
SILVA, L. I., MARTINS, Á. K. L., MAIA, K. K. O., OLIVEIRA, F. B. \& SOUZA, Â. M. A. (2014). Experiências dos familiares de pessoas com transtorno mental. Rev Rene, 15(2), 316-25.

SILVA, T. C. R. \& CAMPOS, M. M. (2015). Gestão de Saúde Mental em Município de Pequeno Porte no Estado do Rio de Janeiro. VÉRTICES, Campos dos Goytacazes/RJ, 17 (3),35-64.

VOSGERAU, D. S. R. \& ROMANOWSKI, J. P.(2014). Estudos de revisão: implicações conceituais e metodológicas. Revista de Diálogo Educacional,(14)41, 165-189.

Submetido em: 29-10-2018

Aceito em: 31-5-2019 